Evangelia ADAMOU

\title{
LE MARQUAGE DIFFERENTIEL DE L'OBJET \\ EN NASHTA ET EN POMAQUE (SLAVE, GRÈCE)
}

Revenir sur l'hypothèse du contact de langues

RÉSUMÉ. - Le marquage différentiel de l'objet (Bossong 1985 ; Lazard 2001) est un phénomène typologique assez courant et bien décrit dans les langues du monde. L'application de ce cadre d'analyse à des faits slaves méridionaux (Adamou 2006) apporte un éclairage nouveau: 1. Elle permet une analyse unifiée de ce phénomène syntaxique commun, quoique exprimé par des moyens différents : par une unité casuelle (génitif-accusatif, ex. pomaque), par une unité préposée (na ex. nashta), voire les deux (ex. macédonien d'Ohrid, Struga) 2. Elle permet d'établir un lien entre ce phénomène dans les variétés contemporaines et en vieux-slave (génitif-accusatif cf. Meillet 1897). 3. Cette approche affaiblit l'hypothèse du contact de langues qui a été avancée pour comprendre «la construction de l'objet direct par la préposition na" (Koneski, Vidoeski \& Jašar-Nasteva 1968 ; Cyxun 1981 ; Topolinjska 1995 ; Markovik 2007 ; Sobolev 2008). Une hypothèse encore plus fragilisée lorsqu'on applique les méthodes des études de contact de langues (Thomason 2001 ; Matras \& Sakel 2007). Cette analyse est illustrée par des données inédites de deux variétés slaves de Grèce (nashta, pomaque).

Dans cet article je présente une approche unifiée de phénomènes observés dans les variétés slaves du sud dont les liens ne sont pas signalés dans la littérature scientifique existante. Je soutiens notamment que le « génitif-accusatif» et ce qui est traditionnellement analysé comme "une construction d'objet direct par une préposition » dans certains parlers slaves méridionaux, sont en réalité l'expression du même phénomène, à savoir le marquage différentiel de l'objet (Bossong 1985 ; Lazard 2001). J'illustre les deux cas de figure par des données inédites à partir de mes enquêtes ${ }^{1}$ sur le nashta et le pomaque de la région de Xanthi, vernaculaires slaves parlés en Grèce.

Dans la tradition des études balkaniques, on parle de «construction avec préposition na pour l'objet direct » (Koneski, Vidoeski \& Jašar-Nasteva 1968 ; Sobolev 2008), "construction accusative avec na » (Topolinjska 1995). On observe alors que "ce phénomène syntaxique n'appartient pas, à proprement parler, au fond des parlers bulgares ${ }^{2} \gg$ (Asenova \& Aleksova $2008: 1$ ).

L'approche que je propose pour analyser les faits slaves méridionaux se focalise sur le phénomène syntaxique de marquage différentiel de l'objet (Adamou 2006 : 64) avec des répercussions importantes sur l'analyse des faits :

\footnotetext{
${ }^{1}$ Enquêtes entre 2002-2004 pour le nashta, puis de 2005-2007 pour le nashta et le pomaque avec le soutien financier du Lacito-CNRS.

${ }^{2}$ Les auteurs suivent ici la position traditionnelle de la dialectologie bulgare qui considère que toutes les variétés au sud du serbe sont des dialectes du bulgare. Toutefois, la standardisation du macédonien après la Deuxième Guerre mondiale et l'indépendance de l'Ancienne République Yougoslave de Macédoine ont consolidé l'existence d'une langue macédonienne et de ses dialectes, approche largement acceptée dans la littérature internationale. Le lecteur doit donc garder à l'esprit que les mêmes dialectes sont nommés tantôt 'bulgares' tantôt 'macédoniens' selon le point de vue national des auteurs.
} 
1. Elle permet de montrer que le marquage différentiel de l'objet est un fait syntaxique commun aux parlers slaves méridionaux, même s'il est exprimé par des moyens différents. En effet, les études typologiques signalent l'expression du marquage différentiel de l'objet soit par des unités casuelles, soit par des adpositions (Lazard 2001), et c'est ce qu'on observe dans les variétés slaves du Sud concernées :

a) D'une part, le marquage différentiel de l'objet s'exprime aujourd'hui encore par un relateur casuel, $-a$, comme en pomaque.

b) D'autre part, le marquage différentiel de l'objet s'exprime à l'aide d'un relateur préposé, $n a$, comme en nashta.

c) Enfin, dans certaines variétés, on observe un stade de coexistence des deux relateurs, casuel $-a$ et préposé $n a$ : par exemple dans les parlers de Struga et de Ohrid (Markovik 2007 : 79, 82).

2. Cette approche permet également d'établir le lien avec le marquage différentiel de l'objet en vieux slave. On constate en effet que les mêmes critères, à savoir l'humanitude, régissent le marquage différentiel de l'objet en vieux-slave et dans les systèmes modernes. Les moyens sont soit les mêmes, à savoir emploi du cas traditionnellement connu comme génitif-accusatif, soit nouveaux, à savoir emploi de la préposition $n a$.

Deux arguments sont avancés pour soutenir le maintien du critère de l'humanitude exprimé par une unité casuelle puis par une adposition :

- L'expression d'une fonction par des unités casuelles est couramment reprise par des adpositions lors du passage d'un système synthétique à un système analytique. Le stade intermédiaire observé à Ohrid et à Struga serait même son illustration.

- La distinction animé-inanimé traversait profondément le vieux-slave comme l'observe Meillet (1897). Il n'est pas surprenant par conséquent qu'elle se soit maintenue lors du passage d'un système synthétique à un système analytique.

L'analyse diachronique semble alors expliquer le mieux les faits dans les variétés contemporaines.

Par ailleurs, je reviens sur l'hypothèse de contact de langues qui a été avancée pour expliquer l'expression de «l'accusatif par la préposition na par de nombreux chercheurs : cf. Koneski, Vidoeski \& Jašar-Nasteva 1968 ; Cyxun 1981 ; Topolinjska 1995 ; Markovik 2007; Sobolev 2008. Avant de développer cette analyse au chapitre 4 , je présente la situation dialectologique et les conditions sociolinguistiques de ces variétés peu connues ( $\$ 1$ et 2). Puis, j'expose le cadre typologique du marquage différentiel de l'objet et les données du nashta et du pomaque $(\S 3)$.

\section{LES VERNACULAIRES SLAVES DE GRECE}

Les variétés vernaculaires slaves du sud sont désignées par de nombreux chercheurs comme dialectes bulgaro-macédoniens (Mazon \& Vaillant 1938, Drettas 1999), par opposition à d'autres groupes slaves méridionaux comme l'ensemble serbo-croate et le slovène. Certains dialectes de cet ensemble slave ont fourni les bases de la langue officielle de Bulgarie, le bulgare littéraire, et plus récemment de la langue officielle de l'Ancienne République Yougoslave de Macédoine (ARYM), le macédonien littéraire.

Les dialectologues répartissent traditionnellement l'ensemble en dialectes occidentaux et orientaux, séparés par ce qu'on appelle la jatova granica, la « limite du jat ». Il s'agit d'une différenciation d'ordre phonétique ; l'ancien ě ayant évolué 
en $e$ dans les dialectes occidentaux, alors que dans les dialectes orientaux $e$ et $j a$ alternent selon les contextes. Selon cette catégorisation traditionnelle le nashta fait partie des dialectes transitoires et le pomaque des dialectes orientaux.

Bien sûr les critères morphosyntaxiques rendent une situation plus complexe : le nashta serait par exemple classé dans l'ensemble occidental, car il a participé à l'innovation d'un parfait avec «avoir», tout en signalant comme spécificité que le parfait avec "être " y a disparu, alors qu'il s'est spécialisé en macédonien pour le médiatif. De même, alors que l'ensemble a participé à l'innovation de l'article défini, se distinguant des autres langues slaves, une distinction s'impose selon que le système des définis s'organise ou non en fonction de la deixis. Cette répartition ne suit pas la ligne de partage est-ouest: ainsi, le nashta emploie un seul défini, comme le groupe oriental, alors que le pomaque dispose d'un triple défini (en fonction de la deixis) le rapprochant du groupe occidental. Enfin, alors que l'ensemble méridional se distingue du serbo-croate par la perte du système casuel, plusieurs variétés de pomaque le conservent à un degré important et avec nombre d'innovations dans ses emplois.

Pour nommer les variétés slaves de Grèce j'ai opté pour les autodénominations (cf. Adamou \& Drettas 2008) : les Pomaques de Grèce nomment leur langue pomatsko "pomaque»; dans le village de Liti c'est 'našta, littéralement « la nôtre » qui a la préférence des locuteurs. Des appellations de ce type ne sont pas rares et soulignent l'attachement des locuteurs à l'identité locale. Par exemple dans le cas de variétés slaves parlées en Italie, Breu (2005: 111) signale qu'une des manières de nommer le parler local est aussi construit sur cette base possessive : na-našu (Acquaviva, San Felice) ; na-našo (Montemitro) « à la nôtre ».

\section{CORPUS ET SITUATION SOCIOLINGUISTIQUE}

En Grèce, tant les variétés slaves des chrétiens que celles parlées par des musulmans sont actuellement en voie de disparition: les chrétiens les ont abandonnées au profit du grec et les musulmans (à l'exception de certains villages) au profit du turc (cf. Adamou \& Drettas 2008).

Le nashta est une variété slave méridionale parlée dans le bourg de $\mathrm{Liti}^{3}$ (appelé Aivati pendant l'Empire ottoman), situé à $10 \mathrm{~km}$ au nord de Salonique, en Grèce. La présence des slavophones dans le village est attestée dans une source byzantine du VII ${ }^{\mathrm{e}}$ siècle (Lemerle $1979: 220 ; 1981$ : 127) et, malgré de nombreux mouvements de populations, leur présence semble avoir été continue jusqu'à nos jours.

Les énoncés examinés proviennent d'un corpus recueilli entre 2002 et 2007 auprès d'une trentaine de locuteurs, nombre d'entre eux étant décédés depuis. Il s'agit de discussions libres et de récits provoqués pour les besoins de l'enquête étant donné que le nashta n'est plus parlé au quotidien dans ce bourg. Les derniers locuteurs compétents sont tous bilingues grécophones-slavophones: les informateurs fluides (nés entre 1918 et 1935) et les plus jeunes (nés entre 19361950) ne pouvant s'exprimer que brièvement en nashta.

En ce qui concerne le pomaque, étant donné les tensions politiques concernant la slavophonie au sein de la minorité musulmane de Grèce, j'ai préféré ne pas citer le nom du village d'enquête, situé dans le département de Xanthi. Dans ce village, les locuteurs maintiennent la variété slave vernaculaire et la transmettent aux enfants contrairement à d'autres villages où le passage au turc est bien avancé.

\footnotetext{
${ }^{3}$ Sur Liti (Letè) voir aussi Tabula Imperii Romani $K$ 34, p. 78 qui atteste l'existence d'un demos de Letè (117 av. J.-C.), habitants de Mygdonie, population d'origine thrace, en partie les Edones. Sur Letè comme évêché voir Oikonomides $1968: 44$, notes et 1.4 et 28.
} 
En général, les enfants apprennent le grec et le turc au moment d'aller à l'école (4-5 ans). Ils suivent également un enseignement religieux (en turc) pour l'apprentissage du Coran (en arabe). Les femmes de plus de 50 ans ont une compétence en grec et en turc leur permettant de tenir une conversation minimale. Les hommes, même âgés, ont une compétence plus importante en grec, grâce au service militaire et aux expériences professionnelles à l'extérieur du village.

Le corpus du pomaque provient de récits, dialogues et énoncés naturels produits spontanément, d'une vingtaine de locuteurs. Ce nombre relativement limité, compte tenu la vitalité de la langue, est lié aux difficultés d'enquête dues à l'enjeu politique que représente le pomaque au sein de la minorité musulmane.

Carte : Le nashta de Liti et le pomaque de la région de Xanthi

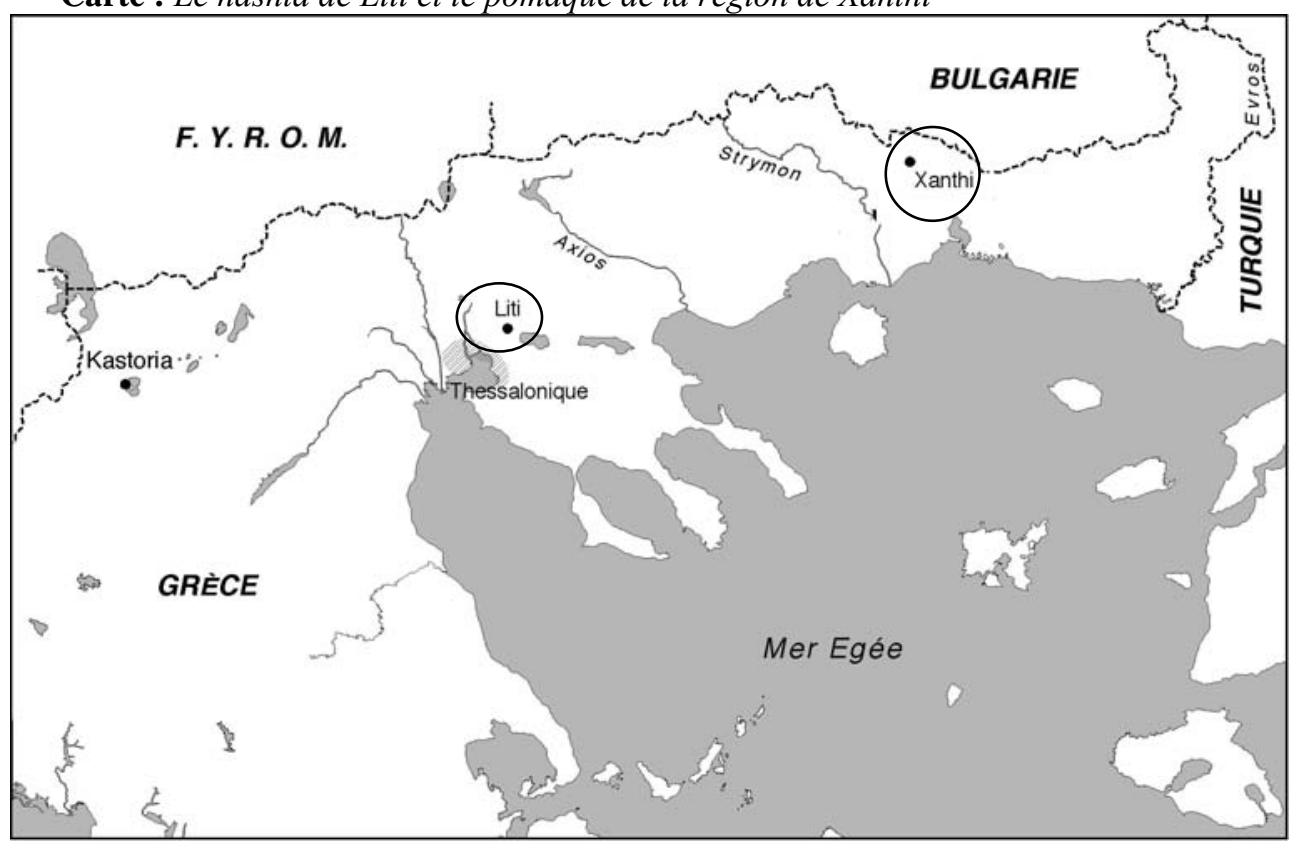

\section{LE MARQUAGE DIFFERENTIEL DE L'OBJET}

\subsection{APPROCHE TYPOLOGIQUE}

Le fait que l'objet direct puisse apparaître dans une langue avec ou sans marque spécifique est assez fréquent dans les langues du monde et on l'appelle differential object marking suite à Bossong $(1985,1998)$; en français marquage différentiel de l'objet suite à Lazard (2001). Selon Lazard l'objet est défini comme l'actant représentant le patient dans une construction biactancielle et tout actant traité de même dans cette construction (Lazard 2001 : 874).

Cette marque spécifique à l'objet direct peut être une unité casuelle ou une unité préposée ou postposée, s'opposant à un objet à cas zéro ou à l'absence d'adposition. Voici un exemple en espagnol avec l'emploi d'une unité préposée :

\section{1. busco mi sombrero}

« Je cherche mon chapeau ».

\section{2. busco a mi amigo}

« Je cherche mon ami ». 
En badaga, langue dravidienne d'Inde, le marquage se fait par une unité casuelle, traditionnellement nommée accusatif, que Pilot-Raichoor (1994) nomme relateur accusatif:

3. ama ondu manusa.na noodida

lui un homme. $\mathrm{RL}^{\mathrm{A}}$ voir $+\mathrm{T} 2+3$

« Il a vu un homme ». (Pilot-Raichoor 1994 : 364).

L'étude des facteurs qui déterminent l'apparition de la marque est assez complexe. Toutefois, les chercheurs s'accordent pour dire que les critères qui régissent le marquage différentiel de l'objet dans les langues du monde sont d'ordre sémantique (humanitude, définitude, sémantisme du verbe) et pragmatique (thématicité, rhématicité).

L'humanitude peut être conçue comme un continuum avec deux pôles, allant du plus humain au plus abstrait. D'après Lazard (1984), le plus humain correspond au locuteur lui-même (EGO), exprimé par les pronoms de $1^{\text {re }}$ et $2^{\mathrm{e}}$ personne, suivi des pronoms de $3^{\mathrm{e}}$ personne et des noms propres, d'un syntagme nominal avec référent humain, non-humain (animé, inanimé) pour aboutir au pôle du moins humain qui inclut le massif (ex. « sable», « eau ») et l'abstraction («liberté », « certitude »). Toutefois l'essentiel est la manière dont le locuteur considère ces entités (Lazard 1994).

Lazard (1994) propose une échelle de définitude qui va d'un pôle plus défini, (présence d'un défini « le ») à un pôle indéfini non référentiel (« un, du») via un indéfini référentiel ( « un certain, un des »).

Enfin, le paramètre d'individuation proposé par Lazard (1984), combine les deux critères d'humanitude et de définitude :

Tableau 1 : Combined scales of definiteness and humanness (Lazard 1984 : 283)

\begin{tabular}{|c|c|c|c|c|c|}
\hline 1 & 2 & 3 & 4 & 5 & 6 \\
\hline $\begin{array}{c}\text { 1st-2nd person } \\
\text { pronouns }\end{array}$ & 3d person pronoun & Definite & Indefinite & Mass & Generic \\
\hline A & Proper names & Human & Non-human & \\
\hline \multicolumn{6}{|r}{ B } \\
\hline
\end{tabular}

Pottier (1968: 87) dans son étude de l'espagnol met l'accent sur la combinaison entre l'objet et l'efficience du verbe, qui peut varier selon les emplois en discours. Il propose donc de considérer deux axes sémantiques, un nominal et un verbal.

\subsection{LES FAITS EN POMAQUE ET EN NASHTA}

Les deux variétés slaves étudiées ici en détail n'emploient pas les mêmes moyens pour exprimer le marquage différentiel de l'objet. Le pomaque l'exprime par un relateur casuel et le nashta par un relateur préposé. Par ailleurs, le marquage différentiel de l'objet est plus développé en nashta qu'en pomaque. J'ai opté pour la glose $2^{\text {ème }}$ participant en nashta, qu'il faut comprendre comme $2^{\text {ème }}$ participant humain. J'ai retenu le terme « accusatif » pour le pomaque.

Le nashta, exprime la fonction objet direct humain par le relateur $n a$, préposé au nom :

4. 'jisk-at da a-'sfalj-at na 'moma-ta

NAS vouloir-3PL MOD 3SG.F.ACC-descendre-3PL 2PART fille-DEF

« Ils veulent faire descendre la fille ». $(\mathrm{H} 1,1924)$ 
L'objet direct non-humain ne reçoit pas de marque, donc pas de préposition $n a$.

Dans la variété pomaque étudiée (région de Xanthi, Grèce), le marquage différentiel de l'objet se manifeste ainsi :

- L'emploi de la marque, $-a$, l'ancien génitif-accusatif, pour la fonction objet direct lorsque l'objet est humain (qu'il soit masculin ou féminin).

- Une marque zéro, tout comme le nominatif, pour l'objet direct nonhumain.

5. huse'in i'ftja-Ø meri'em-a

PMK Hussein.NOM vouloir-AOR.3SG Meriem-ACC

$$
\begin{aligned}
& \text { a'la meri'em gu ni i'ftja- } \\
& \text { mais Meriem.NOM 3SG.ACC NEG vouloir-AOR.3SG } \\
& \text { « Hussein désirait Meriem, mais Meriem ne le désirait pas ». (F1, 1990) }
\end{aligned}
$$

L'accusatif humain - $a$ est également employé avec des prépositions : sas « avec » (ex. sas huseina), $u$ « chez » (u meriema).

L'accusatif non-humain en pomaque de la région de Xanthi s'oppose :

- au nominatif employé pour la fonction sujet :

ex. 'mojet bu'bajk-o 'be Se mi'htar « mon père était mihtar ${ }^{4} »$;

- au datif-génitif employé pour la fonction objet indirect et la possession régie par un verbe :

ex. 'vikam ni'hat-u «j'ai dit à Nihat», 'davam t Soko'lata dje'tine-m « je donne du chocolat aux enfants », pak'bjexa bu'bajk-u « ... mais ils étaient du père»;

Le syncrétisme entre datif et génitif, en la forme de l'ancien datif, que montre ce parler est un développement rare, mais d'une part il s'agit d'un trait commun de l'aire balkanique et d'autre part, on observait déjà en vieux-slave des emplois d'un datif possessif qui tendait à remplacer le génitif (Vaillant $1964: 189$ ).

- ce qu'on catégorise traditionnellement comme un cas mais qui est comme on admet généralement une opération énonciative, le vocatif ex. bu'bajko.

Tableau 2 : Les cas du masculin en pomaque

\begin{tabular}{lll}
\hline NOM & bu'bajko & bu'bajkove \\
\hline GEN-DAT & bu'bajku & bu'bajkovem \\
\hline ACC & bu'bajka & bu'bajkove \\
\hline
\end{tabular}

\subsubsection{HUMANITUDE}

L'analyse des emplois du marquage différentiel de l'objet en pomaque et en nashta révèle la pertinence du critère de l'humanitude.

Dans l'axe de l'humanitude, l'objet direct est marqué dans les cas suivants :

\section{- Noms propres}

Les noms propres ${ }^{5}$ sont systématiquement marqués en pomaque, qu'ils soient masculins ou féminins :

\footnotetext{
${ }^{4}$ Responsables des relations de la commune avec les étrangers.

5 J'ai retenu ici que deux noms très courants (Meriem, Hussein) pour les exemples qui contenaient en réalité des prénoms divers.
} 
$\begin{array}{lllll}\text { 6. } & \text { u'oti } \quad \text { xi } & \text { je } & \text { ne'Jjala } & \text { meri'em-a } \\ \text { PMK } & \text { car } & \text { 3SG.F.DAT AUX.3SG } & \text { trouver.PRF.3SG.F } & \text { Meriem-ACC } \\ & \text { «... car elle lui a trouvée Meriem ». (F1, 1990) }\end{array}$

7. nasra'din-a nje 'rukale na 'sfadba-na

PMK Nasreddin-ACC AUX.3PL NEG appeler.PRF.3PL à mariage-DEF.D « Ils n’ont pas invité Nasreddin au mariage ». (H1, 1935)

8. u'oti 'vika ubi'sljeda meri'em-a

PMK car dire.3SG espionner.3SG Meriem-ACC

« Parce que dit-elle il espionne Meriem ». (F2, 1939)

9. $t i$ 'gali huse'in-a

PMK 2SG aimer-IMP.2SG Hussein-ACC

«Toi, aime Hussein ! » (F2, 1939)

Le génitif-accusatif - $a$ n'était pas employé pour des féminins en vieux-slave ni dans les autres langues slaves à l'exception d'un féminin pluriel qui s'est développé dans certaines langues slaves. Cet emploi observé en pomaque pousse à poser la question de l'origine de la marque : s'agit-il de la même marque que pour les animés masculins, donc y aurait-il une innovation dans ce parler ? Ou bien est-il question d'une évolution phonétique de l'accusatif des noms féminins, le $o$ nasal, qui se serait développé en - $a$, aboutissant ainsi à une homophonie des deux unités ? $\mathrm{Si}-a$ était de nature phonétique, il serait sensible à l'accentuation et se réaliserait différemment en position accentuée et non accentuée. Or, l'examen des données montre que - $a$ est employé tant pour les prénoms féminins accentués que pour les prénoms non-accentués à la dernière syllabe. Cela permet de postuler que cette unité casuelle - $a$ ne résulte pas d'une évolution phonétique de l'accusatif féminin du vieux slave. Reste que, même si il s'agit d'un processus de convergence phonétique de deux cas initialement distincts, l'état actuel de la langue ne permet pas de les distinguer dans leurs usages actuels.

En nashta la marque du deuxième participant humain porte clairement sur tous les prénoms, féminins ou masculins, ainsi que sur les noms de famille.

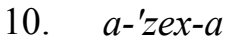
na 'sul-ta 'netre
NAS 3SG.F.ACC-prendre.AOR-2PL 2PART Sula-DEF dedans « Ils ont mis Sula en prison ». (F1, 1924)

Probablement sous l'influence du grec, le nom propre en nashta est souvent déterminé par un défini :

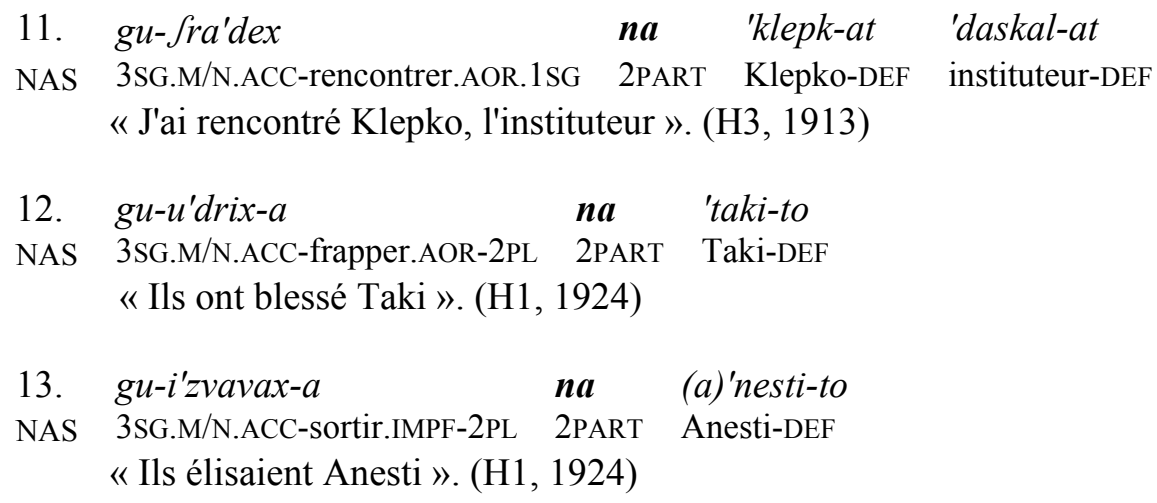


14. na po'stoli gu-'platSax-me

NAS 2PART Apostoli 3SG.M/N.ACC-pleurer.IMPF-1PL

« Nous étions en train de pleurer Apostoli ». (F2, 1922)

- Noms faisant référence à des êtres humains

En nashta, tous les noms référant à des humains reçoivent la marque, y compris des noms souvent traités à part dans les langues du monde (ex. badaga) et dans la région, ex. la fille :
15. $u$-ka'lde
na zet
'jimafe
zet
NAS 3SG.M/N.ACC-mettre.AOR.3SG 2PART gendre avoir.IMPF.3SG gendre «Elle a mis le gendre (dans l'entreprise), il y avait un gendre ... » (H2, 1927)
16. i a-u'stava na 'moma-ta
NAS et 3SG.F.ACC-laisser.IMPF.3SG 2PART fille-DEF
«... et il quitte la fille ». $(\mathrm{H} 2,1927)$

Signalons qu'en nashta les fantômes ${ }^{6}$ sont traités comme des humains et reçoivent la marque systématiquement :

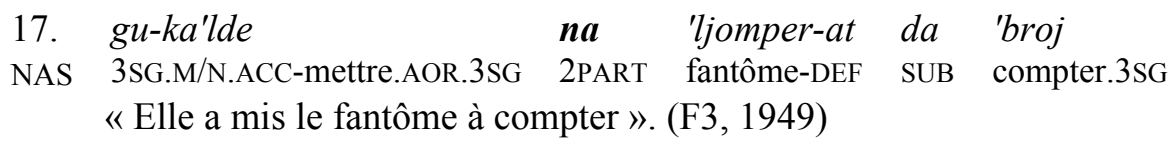

En pomaque, le marquage des humains est très restreint et se limite à certains noms de parenté, comme ici le père, figure importante dans l'aire balkanique dont le nom est souvent traité à part :

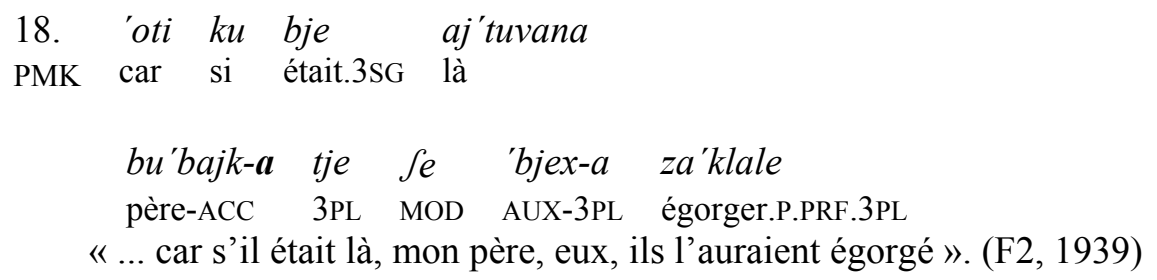

On le retrouve aussi avec 'djado-'djada " grand-père ", alors que pour les féminins en - $a$ ('baba " grand-mère ", 'majka "mère ", 'abla " soeur aînée » etc.) il n'y a pas de distinction à l'accusatif. Toutefois, dans des variétés voisines, les féminins en $-a$, ex. zho'na «femme» forment un accusatif en -o, ex. 'zhono, probablement du à un développement phonétique (cf. Kokkas 2004).

Les autres noms des humains ne présentent pas cette marque - $a$ : ex. 3e'na « femme », tfu'ljak « homme », duo'ktor « docteur », a'fkatin « avocat», etc.

\section{- Noms faisant référence à des objets animés}

En nashta, le marquage d'un nom qui réfère à un objet animé dépend du contexte d'apparition. Lorsque l'objet animé non humain apparaît dans un contexte habituel il n'est pas marqué. Or, certaines unités dont le référent n'est pas humain

\footnotetext{
${ }^{6}$ Traduit ainsi par les locuteurs mais rejoignant dans la région la tradition de la croyance aux vampires.
} 
peuvent être considérées comme telles dans certains cadres narratifs, comme les contes, et recevoir à ce titre la détermination du fonctionnel $n a$. Ainsi, des animés qui sont pris pour des humains ou des fantômes reçoivent la marque. Dans ce conte, le fantôme croit que la petite-fille s'est transformée en chèvre :

$$
\begin{array}{llll}
\text { 19. } & a-' t \int u j & \boldsymbol{n a} & \text { 'kos-ta } \\
\text { NAS } & \text { 3SG.F.ACC-entendre.3SG } & \text { 2PART } & \text { chèvre-DEF } \\
& \text { « Il entend la chèvre ... » } & (\mathrm{F} 3,1949)
\end{array}
$$

En pomaque, les animés même dans des contextes magiques des contes, ne reçoivent pas le marquage de l'humanitude.

\section{- Noms référant à des objets inanimés}

Le marquage d'un nom qui réfère à un objet inanimé dépend du contexte d'apparition. Lorsque l'objet inanimé apparaît dans un contexte magique il peut se comporter comme une entité magique et être traité syntaxiquement comme un humain. Ainsi quand un arbre s'agrandit lorsque le génie le lèche, afin de protéger la fille qui se trouve dessus, l'arbre sort de sa condition habituelle et dévient, sur ce point du récit, un personnage qui à ce titre nécessite l'emploi du fonctionnel na.

$$
\begin{array}{llll}
\text { 20. i'lent } \int e-t o & g u \text {-'li 3iSe } & \boldsymbol{n a} & \text { 'dəb-at } \\
\text { NAS faon-DEF } & \text { 3SG.M/N.ACC-lécher.IMPF.3SG 2PART } & \text { arbre-DEF } \\
& \text { "Le génie-faon léchait l'arbre }
\end{array}
$$

Dans le même conte, mais dans un contexte où les propriétés magiques de l'arbre ne sont pas évoquées, l'arbre ne reçoit pas la marque, étant considéré donc comme un objet inanimé habituel.

$$
\begin{aligned}
& \text { 21. sa ba'ltak-at da-gu-'satf-et 'dab-at } \\
& \text { NAS avec hache-DEF VOL-3SG.M/N.ACC-couper-3PL arbre-DEF } \\
& \text { « Pour couper l'arbre à la hache ». (H1, 1924) }
\end{aligned}
$$

\section{- Pronoms personnels}

En nashta, le procédé morphologique du marquage d'un objet humain est très clair et porte sur les pronoms détachés du syntagme verbal. Notons que en nashta, les pronoms suffixés au syntagme verbal sont obligatoires pour un objet défini ; il s'agit du phénomène connu comme redoublement clitique que Bossong (1998) propose de considérer comme un marquage différentiel en fonction de la définitude.

Sont employés avec la marque $n a$ tous les deux paradigmes de pronoms détachés : d'une part, ceux qui sont employés pour la mise en valeur et d'autre part, ceux qui s'emploient avec les fonctionnels ( $z a$ «pour», $n a$ «allatif») (Adamou $2006: 33$ ). Signalons que les formes employées pour la mise en valeur sont les mêmes que les génitifs-accusatifs du vieux-slave.

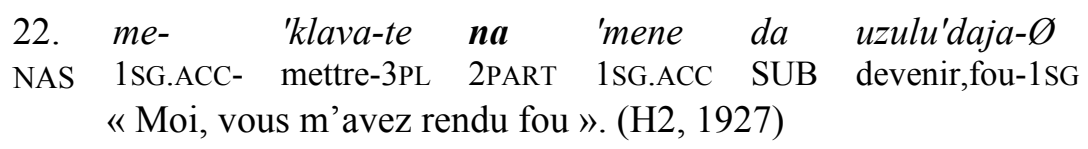

\footnotetext{
${ }^{7}$ La traduction exacte en nashta est bien « arbre » et non le sens spécialisé « chêne ».
} 
23. u-'klava-t na to $d a$ 'bide-Ø de'rvena'gas

NAS 3SG.M/N.ACC-mettre-3PL 2PART 3SG SUB devenir-3SG chef

« Ils l'ont fait devenir chef ». (H2, 1927)

En pomaque, les pronoms personnels ont des formes différentes en fonction sujet, en fonction objet direct (dit accusatif) et en fonction objet indirect (dit datif). Il existe également des formes intensives qui historiquement sont des génitifsaccusatifs. En vieux-slave, les formes intensives sont analysées comme des génitifs-accusatifs qui s'opposent aux formes accusatives (Vaillant 1964 : 179).

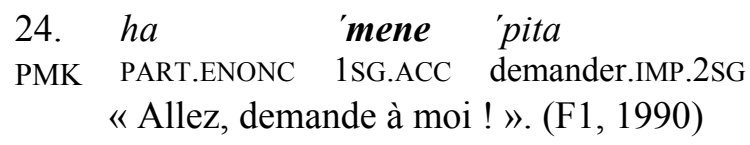

\subsubsection{DEFINITUDE}

Les études sur le fonctionnement du marquage différentiel de l'objet ont permis d'identifier la définitude comme critère régissant ce phénomène, un objet défini recevant une marque spécifique qu'un objet générique ne reçoit pas.

En nashta et en pomaque l'inanimé est relié directement au verbe même lorsqu'il est déterminé par un défini.

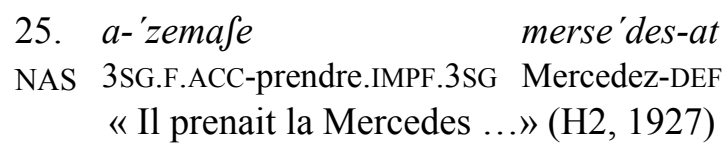

\subsubsection{THEMATISATION}

Les études sur le marquage différentiel de l'objet ont signalé que pour certaines langues un objet thématique est marqué. En nashta ce critère ne semble pas pertinent. L'objet est marqué lorsqu'il est humain ou non humain, qu'il soit thématique ou non. Voici un exemple avec un participant humain en position thématique et rhématique :

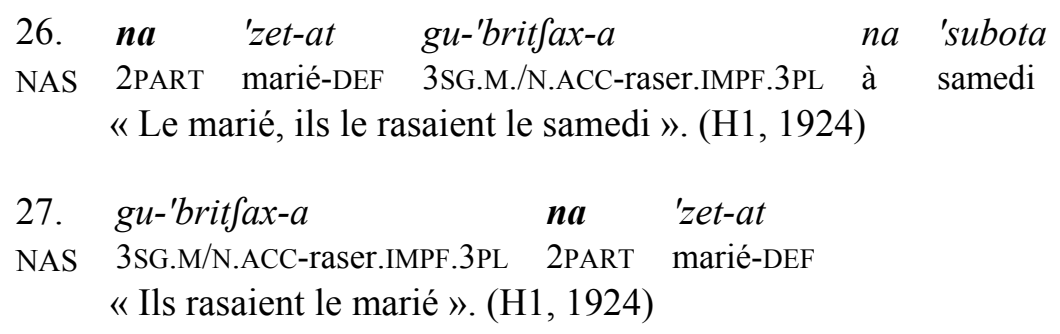


Tableau 2 : L'échelle du marquage différentiel de l'objet en nashta et en pomaque

\begin{tabular}{|c|c|c|c|c|c|c|}
\hline & 1 & 2 & 3 & 4 & 5 & 6 \\
\hline & $\begin{array}{l}\text { pronoms } \\
\text { personnels } \\
1 \mathrm{e}-2 \mathrm{e} \\
\text { personne }\end{array}$ & $\begin{array}{c}\text { pronom } \\
\text { personnel } \\
\text { 3e personne } \\
\text { Noms } \\
\text { propres }\end{array}$ & Humain & $\begin{array}{l}\text { Non-humain } \\
\text { (animé, } \\
\text { inanimé) }\end{array}$ & Massif & Générique \\
\hline & + & + & + & $+/-$ & - & - \\
\hline NAS & $\begin{array}{l}\text { personnels } \\
\text { indépendants }\end{array}$ & $\begin{array}{c}\text { noms } \\
\text { propres } \\
\text { masculins \& } \\
\text { féminins }\end{array}$ & $\begin{array}{c}\text { noms } \\
\text { variés } \\
\& \\
\text { fantôme }\end{array}$ & $\begin{array}{c}\text { animaux, } \\
\text { arbres } \\
\text { personnifiés ou } \\
\text { magiques }\end{array}$ & & \\
\hline PMK & $\begin{array}{l}\text { personnels } \\
\text { indépendants }\end{array}$ & $\begin{array}{c}\text { noms } \\
\text { propres } \\
\text { masculins \& } \\
\text { féminins }\end{array}$ & $\begin{array}{c}\text { termes } \\
\text { de } \\
\text { parenté }\end{array}$ & & & \\
\hline
\end{tabular}

\section{L'HYPOTHESE DE CONTACT DE LANGUES}

L'expression « de l'objet direct par la préposition $n a »$ a été considéré comme un phénomène nouveau dans les variétés slaves du Sud et c'est le contact de langues qui a été évoqué comme facteur explicatif. Ce sont donc les moyens employés pour indiquer l'objet direct qui ont soulevé cette question. Toutefois, je soutiens ici qu'il s'agit de l'expression d'un phénomène syntaxique commun, le marquage différentiel de l'objet (Adamou 2006: 64), bien décrit en vieux-slave, dans les langues slaves et dans de nombreuses variétés orales de l'aire.

Il est par ailleurs utile de réévaluer l'hypothèse explicative de contact, à la lumière des acquis récents dans les études de contact de langues et il convient de se poser les questions suivantes (Thomason 2001) :

\section{Le fait est-il attesté en diachronie?}

La première question avant de qualifier un fait comme résultant d'un contact de langues est de savoir si nous avons des traces de ce phénomène en diachronie. Souvent difficile à traiter dans les aires où l'on manque de documentation écrite, et à aborder avec précaution lorsqu'on dispose d'une documentation écrite - sachant la distance entre les variétés écrites et orales -, cette question reste importante dans l'aire balkanique pour laquelle nous disposons de documents écrits.

\section{1a. Attesté en vieux-slave}

La distinction entre objets humains vs non-humains est bien décrite pour le vieux-slave, la variété de slave écrite la plus ancienne, attestée dans des textes ecclésiastiques de $850-1100$; cf. entre autres Meillet 1897; Vaillant 1977 : 38 ; Huntley 1993 ; Kulikov 2006. En vieux slave on distingue 7 cas au singulier et au pluriel, à savoir nominatif, accusatif, génitif, locatif, datif, instrumental et vocatif. $\mathrm{Au}$ duel on compte seulement nominatif-accusatif, génitif-locatif et datifinstrumental.

La position traditionnelle défend que le syncrétisme entre nominatif et accusatif, suite à la chute des consonnes finales en slave commun, avait crée une ambiguité qui n'était pas tolérée pour certains noms, notamment pour les objets animés qui sont habituellement en fonction sujet. Dans le cas des noms animés, la 
marque du génitif, - $a$, a été employée pour la fonction objet direct ou accusatif. C'est donc ce qui est connu comme génitif-accusatif.

Initialement employé pour les noms de personnes masculins, ce marquage se rencontre dans les textes avec les pronoms mais aussi avec des objets qui sont personnifiés : par exemple "l'enfer », comme dans " Hadès, prince de l'Enfer » (Vaillant 1964: 177; aussi Huntley 1993). De même, les noms d'animaux personnifiés sont employés avec le génitif-accusatif.

Ces facteurs se retrouvent également dans les variétés contemporaines même si nous attestons des emplois innovants, comme par exemple l'emploi avec les prénoms féminins en nashta et en pomaque.

1b. Importance de la distinction humain vs non-humain dans le système

Meillet (1897: 150) reliait au phénomène du marquage différentiel de l'objet, les emplois du génitif possessif et de l'adjectif possessif pour distinguer les noms de personnes des noms d'objets inanimés. En effet, Meillet notait déjà en 1897 que «le génitif possessif est remplacé d'une manière constante [...] par un adjectif dérivé, quand ce génitif est celui d'un substantif indiquant une personne " (Meillet 1897 : 148). Cet élément donne à la distinction humain vs non-humain une place fondamentale dans la langue. C'est cette caractéristique centrale qui nous semble importante à retenir afin de comprendre les cas de figure où la perte du système casuel n'a pas pour autant fait disparaître l'expression différentielle de l'objet.

En pomaque ces emplois sont encore d'actualité. Ainsi, pour un nom propre, un nom de parenté ou un nom se référant à un humain c'est un adjectif possessif (l'adjectif dérivé de Meillet) qui marque la possession :

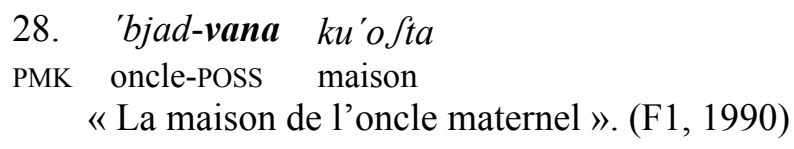

Pour les objets inanimés les locuteurs les plus jeunes emploient majoritairement le fonctionnel $u t$ :

$$
\begin{aligned}
& \text { 29. 'ziume } \quad \text { 'rjesta-na } \text { ut ti'ropita-na } \\
& \text { PMK prendre.IMP.2SG monnaie-DEF.E de feuilleté-DEF.E } \\
& \text { « Prends la monnaie du feuilleté au fromage ». (H1, 1995) }
\end{aligned}
$$

Cette observation nous permet de soutenir que le passage d'un système synthétique à un système analytique peut se faire en gardant la pertinence de la distinction animé-inanimé, voire en se servant de cette distinction pour évoluer, et par conséquent le marquage différentiel de l'objet en fonction de l'humanitude peut survivre à la perte du système casuel.

\section{1c. Les stades d'évolution diachronique illustrés par la variation dialectale}

Comme c'est connu dans les études dialectales, le continuum dans l'espace peut illustrer des stades d'évolution dans le temps :

stade A: les variétés modernes qui emploient le même relateur casuel $a$ qu'on retrouve dans les textes écrits (ex. pomaque);

stade B: les variétés modernes qui emploient le relateur casuel - $a$ et le relateur préposé na (ex. Struga, Ohrid, cf. Markovik 2007);

stade $\mathrm{C}$ : les variétés modernes qui emploient uniquement le relateur préposé na (ex. nashta, cf. Adamou 2006). 
Tableau 3 : L'évolution du marquage différentiel de l'objet dans la variation dialectale

\begin{tabular}{ccc}
\hline & $\Delta+$ & \\
\hline stade A & stade B & stade C \\
\hline relateur casuel & relateur casuel + relateur préposé & relateur préposé \\
$-a$ & $-a+n a$ & $n a$ \\
\hline pomaque & macédonien Struga, Ohrid & nashta \\
& Markovik 2007 & Adamou 2006 \\
\hline
\end{tabular}

\section{1d. Les traces écrites}

Le problème reste bien sûr l'absence de traces écrites des variétés orales qui pourraient démontrer ce passage en détail. Or, il est bien connu que les variétés orales n'étaient que peu écrites. Même dans les textes qui leur étaient le plus proche les analystes craignent l'influence de la langue littéraire, puisque ce sont en général des savants qui écrivent ces textes. Par exemple, dans des textes plus contemporains de l'aire sud-occidentale, comme l'Evangéliaire de Kulakia $\left(19^{\text {ème }}\right)$ (Mazon \& Vaillant 1938) et celui de Konikovo (fin $18^{\text {ème }}$ ) (Lindstedt, Spasov, Nuorluoto 2008), les prénoms masculins présentent la marque - $a$, le génitifaccusatif : ami da vidat $i$ Lazara « ... mais aussi pour voir Lazar ... » (Lindstedt, Spasov, Nuorluoto $2008: 175)$

Or, Mazon et Vaillant (1938: 101) qualifient les emplois de Kulakia comme propres à la langue religieuse : si leur hypothèse est vraie, cela confirme notre incapacité à savoir comment la variété orale était réellement parlée. Si elle est erronée, cela confirme le maintien de ce phénomène dans les variétés orales plus longtemps que ce qu'on croyait.

\section{Le fait est-il observé dans les autres langues slaves?}

Le marquage différentiel de l'objet est un phénomène syntaxique courant dans les langues slaves, exprimé par les unités casuelles. Bossong (1998: 212) distingue deux catégories dans les langues slaves :

I) celles qui ont une flexion casuelle avec MDO à deux étages :

a) Opposition neutre vs. masculin/féminin : catégorie pétrifiée, héritée de I'indoeuropéen.

b) Opposition animé $v s$. inanimé : catégorie vivante comme résultat d'une évolution nouvelle: vieux bulgare, slovène, serbo-croate, polonais, sorabe, tchèque, slovaque, ukrainien, russe et biélorusse.

II) Celles qui ont perdu la flexion casuelle, le bulgare et le macédonien. Pour ces derniers, Bossong se réfère à deux procédés de MDO liés à la définitude : l'article défini masculin en bulgare, et le redoublement de l'objet en macédonien. Il me semble toutefois que les données présentées dans cet article confirment l'emploi du MDO en fonction de l'humanitude dans les variétés orales du Sud. 
Bossong $1998: 213$ ( $\Delta$ = différentialité, $\mathrm{A}=$ accusatif, $\mathrm{N}=$ nominatif, $\mathrm{G}=$ Génitif, $\mathrm{F}=$ féminin, $\mathrm{M}=$ masculin, $\mathrm{SG}=$ singulier, $\mathrm{PL}=$ pluriel, $\mathrm{DU}=$ duel)

(17) Répartition générale du MDO dans les langues slaves I

\begin{tabular}{|c|c|c|c|c|c|c|}
\hline & F SG & F DU & F PL & M SG & M DU & M PL \\
\hline Vieux bulgare & $A \neq N$ & $A=N$ & $A=N$ & $\begin{array}{l}\Delta(A) \\
{[A=G]}\end{array}$ & $A=N$ & $A \neq N$ \\
\hline Slovène & $A \neq N$ & $A=N$ & $A=N$ & $\begin{array}{l}\Delta(\mathrm{A}) \\
{[\mathrm{A}=\mathrm{G}]}\end{array}$ & $A=N$ & $A \neq N$ \\
\hline Serbo-croate & $A \neq N$ & $\emptyset$ & $A=N$ & $\begin{array}{l}\Delta(\mathrm{A}) \\
{[\mathrm{A}=\mathrm{G}]}\end{array}$ & $\emptyset$ & $A \neq N$ \\
\hline Polonais & $A \neq N$ & $\emptyset$ & $A=N$ & $\begin{array}{l}\Delta(A) \\
{[A=G]}\end{array}$ & $\emptyset$ & $\begin{array}{l}\Delta(A, N) \\
{[A=G]}\end{array}$ \\
\hline Haut-sorabe & $A \neq N$ & $A=N$ & $A=N$ & $\begin{array}{l}\Delta(\mathrm{A}) \\
{[\mathrm{A}=\mathrm{G}]}\end{array}$ & $\begin{array}{l}\Delta(A) \\
{[A=G]}\end{array}$ & $\begin{array}{l}\Delta(A, N) \\
{[A=G]}\end{array}$ \\
\hline Tchèque & $A \neq N$ & $\emptyset$ & $A=N$ & $\begin{array}{l}\Delta(\mathrm{A}) \\
{[\mathrm{A}=\mathrm{G}]}\end{array}$ & $\emptyset$ & $\begin{array}{l}\Delta(A, N) \\
{[A \neq G]}\end{array}$ \\
\hline Slovaque & $A \neq N$ & $\emptyset$ & $A=N$ & $\begin{array}{l}\Delta(A) \\
{[A=G]}\end{array}$ & $\emptyset$ & $\begin{array}{l}\Delta(A) \\
{[A=G]}\end{array}$ \\
\hline Ukrainien & $A \neq N$ & $\emptyset$ & $\begin{array}{l}\Delta(A) \\
{[A=G]}\end{array}$ & $\begin{array}{l}\Delta(\mathrm{A}) \\
{[\mathrm{A}=\mathrm{G}]}\end{array}$ & $\emptyset$ & $\begin{array}{l}\Delta(\mathrm{A}) \\
{[\mathrm{A}=\mathrm{G}]}\end{array}$ \\
\hline Biélorusse & $A \neq N$ & $\emptyset$ & $\begin{array}{l}\Delta(A) \\
{[A=G]}\end{array}$ & $\begin{array}{l}\Delta(A) \\
{[A=G]}\end{array}$ & $\emptyset$ & $\begin{array}{l}\Delta(\mathrm{A}) \\
{[\mathrm{A}=\mathrm{G}]}\end{array}$ \\
\hline Russe & $A \neq N$ & $\emptyset$ & $\begin{array}{l}\Delta(\mathrm{A}) \\
{\left[\mathrm{A}=\mathrm{G}_{1}\right]}\end{array}$ & $\begin{array}{l}\Delta(A) \\
|A=G|\end{array}$ & $\emptyset$ & $\begin{array}{l}\Delta(A) \\
|A=G|\end{array}$ \\
\hline
\end{tabular}

\section{Le fait est-il typologiquement courant?}

Pour caractériser un fait comme étant du au contact de langues, Thomason (2001) propose de distinguer entre les faits typologiquement courants, qui peuvent surgir hors contact, et les faits typologiquement rares, plus faciles à identifier comme étant dus au contact de langues. En suivant cette observation j'ai signalé que le processus suivi en nashta pour le marquage différentiel de l'objet est assez banal d'un point de vue typologique (Adamou $2006: 64$ ) et donc l'hypothèse du contact de langues est plus douteuse.

En effet dans ce parler la marque qui est utilisée pour le marquage différentiel de l'objet sert pour l'accusatif, le datif, l'allatif (parfois aussi le locatif) et la subordination: des caractéristiques courantes selon les études typologiques (Heine et al. 1991 : 155). De même en nashta, $n a$ est aussi le fonctionnel de l'objet indirect :

30. 'nosax-a na 'star-te 'mandza

NAS apporter.IMPF-3PL à vieux-DEF nourriture « Ils apportaient de la nourriture aux vieux ».

31. So ki-'berex-a na ne'vasta-ta

NAS quoi INT-apporter.IMPF-3PL à mariée-DEF «Qu'est-ce qu'ils apportaient à la mariée? » 
Par ailleurs, $n a$ connait des emplois comme allatif et comme subordonnant de but (Adamou 2006) :

32. ki-'doja-m na 'kum-to na 'vetfer

NAS INT-venir-1SG ALL/DUR parrain-DEF ALL/DUR soirée

« Je viendrai chez le parrain en soirée ».

$\begin{array}{lllllll}\text { 33. } & d a & \text { 'vikni-s } & \text { na } & \text { ma'ria-ta } & \text { na } & \text { 'doj-Ø } \\ \text { NAS } & \text { MOD appeler-2SG } & \text { 2PART } & \text { Maria-DEF } & \text { SUB } & \text { venir-3SG } \\ & \text { « Appelle Maria qu'elle vienne ». } & & & \end{array}$

Pour ce dernier emploi, qui est assez fréquent, on peut formuler deux hypothèses : l'une est qu'il s'agit d'un emprunt du subordonnant grec $n a$; l'autre qu'il s'agit d'une évolution interne à la langue, suivant des tendances cognitives universelles (Heine et al. 1991).

\section{Attester un contact effectif entre les locuteurs}

Se pose aussi la question de savoir s'il faut qu'il y ait toujours un contact de langues identifiable afin de qualifier un phénomène comme du au contact. Même si cela reste souhaitable on sait qu'il est parfois difficile de le démontrer et souvent dans des aires pour lesquelles on ne dispose pas de données historiques on s'appuie sur les faits linguistiques afin de démontrer les contacts des populations. Il nous semble toutefois que pour des aires pour lesquelles nous avons des connaissances historiques, comme les Balkans, cette question a le mérite de se poser.

Pour expliquer l'emploi de la structure accusative avec $n a$ qu'on avait déjà observé dans les textes de Vatilak (région de Salonique) (Miletič 1932) on évoque l'« influence romane» comme explication (Cyxun 1981). Cette explication est souvent avancée (ex. parfait avec 'avoir) sans pour autant pouvoir démontrer ce contact dans les régions données ni situer les deux chronologiquement.

Par exemple, nous disposons de renseignements assez précis sur les populations aroumaines et les zones de présence. La zone autour du fleuve Axios fait partie des zones de pâturage d'été mais l'aire autour de Salonique n'y est pas concernée, à l'exception de Oreokastro avec une faible population aroumaine (Trifon 2005). Nous ne pouvons pas non plus attester historiquement d'un bilinguisme ou d'un contact intense du nashta avec l'aroumain. Pour ce qui est de la fin $\mathrm{du} \mathrm{XIX}^{\mathrm{e}}$ siècle, nous savons que la population du village était à forte majorité slavophone. Un des rares chants de mariage du début du $\mathrm{XX}^{\mathrm{e}}$ siècle dont se souviennent les derniers locuteurs dit précisément :

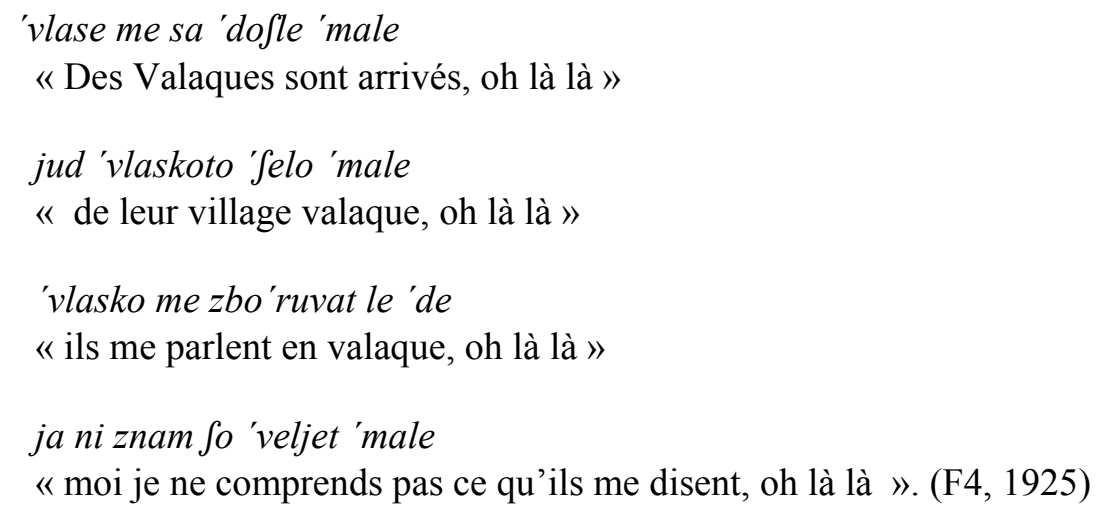

Ce chant indique donc les contacts avec des Valaques, venus de leur village, mais met plutôt l'accent sur l'absence de connaissance du valaque. Les derniers locuteurs se souviennent d'une famille valaque dans village, fait très périphérique. 
Il est évident que plus nous remontons loin dans l'histoire, plus les contacts sont difficiles à saisir. Nous savons par exemple qu'au cours du XIX ${ }^{\mathrm{e}}$ siècle des immigrés venus d'autres villages se sont installés à Aivati et qu'ils ont même fait partie des élites (enquête personnelle). Nous ne savons pas pour autant si ils étaient de langue maternelle slave ou autre. Karakasidou (2000) dans son étude du village d'Assiros (gr.) / Giouvezna montre bien comment les hommes immigrés épousaient des femmes slavophones et elle confirme leur connaissance du slave local. On peut bien entendu envisager un scénario où ces personnes socialement importantes et influentes ont pu être à l'origine de la généralisation de certains traits non slaves dans le vernaculaire local : on peut imaginer qu'ils aient intégré certains traits de leur langue d'origine dans leur manière de parler le slave. Il n'est pas impossible que ces innovations, aient trouvé leur place dans le système du slave local, puis aient été reprises et généralisées par différentes fractions de la population. Toutefois, à défaut d'éléments de preuve, cette hypothèse doit être traitée avec précaution et en lien avec les autres facteurs examinés plus haut.

\section{Si contact de langues attesté : attester l'emploi du trait dans la langue source}

Pour les variétés qui se situent dans une zone de contact attesté avec l'aroumain l'hypothèse du contact de langues devient plus plausible. On évoque dans ce cas le contact à l'aroumain : Kastoria (gr.) / Kostur, Kajlar et Debar, cf. Koneski, Vidoeski \& Jašar-Nasteva (1968). Toutefois, même si le roumain dispose d'une préposition pe pour l'objet direct, certains parlers aroumains de la région ne disposent pas de ce trait: ex. Gołab (1984) pour Krushevo ; Beis (2000) pour Metsovo.

Ce n'est que très récemment, à la lumière de nouvelles données qui signalent son existence dans certains parlers aroumains de la région, que l'hypothèse du contact à l'aroumain revient à l'ordre du jour. Markovik (2007) signale son emploi en aroumain Frasherot de la région d'Ohrid ; Bara, Kahl \& Sobolev (2005) notent son usage pour Krania (gr.) / Turya, sous les formes pri, pra, pi, pe (variantes phonétiques) : ați'ol al'and" u ak'ațā prā mul"ari-sa « et un autre homme prend sa femme ... » (Bara, Kahl \& Sobolev $2005: 44)$.

Pour Sobolev (2008) constater l'existence de ce phénomène dans certaines variétés aroumaines de la région et dans certaines variétés slaves de la région suffit à démontrer le scénario du contact de langues. Or, on manque de données, tant sur les variétés slaves que sur les variétés aroumaines, afin de pouvoir tracer ce phénomène. Il semblerait qu'il existe plus de variétés, slaves et aroumaines, qui ont des emplois de ce type que ce qu'on croyait jusque là : parmi les variétés slaves, celle de Hrisa (c.p. Drettas) qui ne fait pas partie des 'zones' connues jusque là ; parmi les variétés aroumaines, on en trouve certaines qui n'ont pas eu de contact au roumain (ex. via les écoles roumaines) cf. Kokka (1992:18).

D'autres hypothèses de contact ont été avancées pour essayer d'expliquer cette originalité morphologique de ces variétés slaves. Topolinjska (1995: 95) signale une origine grecque. Or, les variétés grecques n'expriment pas le marquage différentiel de l'objet, à l'exception notable des variétés cappadociennes en fonction de la définitude (cf. Janse 2004 où il argumente pour le contact au turc).

\section{Si emploi du trait dans la langue source : observer le résultat sur la langue d'accueil (calque ou emprunt)}

Les études sur le contact de langues s'intéressent aux résultats observés dans la langue d'accueil suite au contact de langues : ainsi Heine \& Kuteva (2005), suite à Weinreich (1953) distinguent les cas de replication des cas de borrowing. Matras \& Sakel (2007), tentent de comprendre le mécanisme de replication en le reliant à 
des facteurs structurels et sociolinguistiques. Ainsi, ils discutent des catégories qui se prêtent plus aisément au calque grammatical ou à l'emprunt.

Mladenov (1993) signale l'emprunt de la préposition pe en bulgare au contact du roumain : téjku jŭ zé pŭ máminŭtŭ májkŭ « Le père a pris la mère de la mère avec lui ». (Mladenov $1993: 381$ )

Cet exemple est traditionnellement cité comme une preuve du scénario de contact entre les variétés macédoniennes et l'aroumain. Or, les données de Mladenov confirment seulement la possibilité qu'une langue slave puisse être influencée par le contact avec une langue romane sur le point du marquage différentiel de l'objet.

Néanmoins, elles posent la question de l'effet du contact de langues: pourquoi au cas du bulgare nous avons un emprunt au roumain, alors qu'au cas du macédonien un calque à l'aroumain ? Cette différence n'est jamais relevée, or elle semble importante à explorer. Quelles sont les caractéristiques structurelles ou sociolinguistiques (ex. conditions de bilinguisme) qui expliquent la divergence des résultats? Pourquoi les variétés de macédonien n'ont pas également emprunté à l'aroumain la préposition pe pour ces emplois ? Ce travail serait intéressant à mener me semble-t-il si l'on veut soutenir l'hypothèse du contact de langues, qui se matérialise avant tout comme un contact entre locuteurs.

\section{Si calque : chercher le point 'pivot'}

Des études comme celle de Markovik (2007) sont précieuses pour notre compréhension du phénomène. Markovik signale en effet ce phénomène pour des locuteurs bilingues d'aroumain Frasherot d'Ohrid et de macédonien d'Ohrid, donc avec un contact de langues attesté :

\section{4. a. Macédonien d'Ohrid : go vidov na t Sovekot \\ b. Aroumain Frasherot d'Ohrid : u va 3dui pi omu « J'ai vu l'homme». (Markovik 2007)}

Or, il me semble que pour soutenir que l'emploi du macédonien est un calque à l'aroumain il faudrait que ces unités partagent d'autres emplois communs qui auraient pu servir de "pivot» (Matras \& Sakel 2007). En effet, si deux langues en contact disposent de deux unités servant pour la fonction $x$ alors on peut penser que la fonction $z$ assumée par l'unité dans la langue modèle (ML), mais non dans la langue de contact, puisse être calquée (RL replica language). Toutefois, ce point pivot n'est pas évident à repérer pour le macédonien et l'aroumain : outre l'objet direct humain, $n a$ sert en macédonien pour la fonction objet indirect et comme locatif, alors que $p i$ sert en aroumain comme locatif. C'est une autre préposition, $a l$, qui sert pour l'objet indirect :

\section{5. a. Macédonien d'Ohrid : my rekov na $t$ Sovekot}

b. Aroumain d'Ohrid : lji spus al omu

« J'ai dit à l'homme ». (Markovik 2007)

Markovik (2007 : 92) s'appuie sur le partage des emplois locatifs pour démontrer le calque des emplois accusatifs. Toutefois, il ne nous parait pas convaincant que le locatif puisse servir de pivot dans ce cas. On s'attendrait plutôt à ce que la fonction d'objet indirect soit le pivot, puisqu'elle s'inscrit aussi dans la sphère des participants au procès ; or, celle-ci n'est pas exprimée en aroumain par $p i$ mais par al (35b).

En regardant le tableau ci-dessous on se rend compte que l'emploi de $n a$ « objet direct» en macédonien d'Ohrid a plus de chances d'avoir été influencé par 
l'emploi de $n a$ « objet indirect» dans la langue même, que du parallélisme établi entre le $p i$ « locatif» de l'aroumain et le $n a$ « locatif » du macédonien.

Tableau 4 : Les emplois de «na» en macédonien d'Ohrid et de «pi» en aroumain d'Ohrid

\begin{tabular}{lccc}
\hline & LOCATIF & $\begin{array}{l}\text { OBJET DIRECT } \\
\text { (HUMAIN) }\end{array}$ & OBJET INDIRECT \\
AROUMAIN D'OHRID & pi & pi & al \\
MACÉDONIEN D'OHRID & na & na & $<=$ na \\
\hline
\end{tabular}

Il est certain qu'un cas de bilinguisme attesté, tel qu'il est décrit par Markovik, exerce sans doute une influence sur la vitalité de ce phénomène. Toutefois, force est de constater que même dans des régions dans lesquelles ce type de bilinguisme n'est pas attesté, comme pour le nashta de Liti, le phénomène et l'emploi de la préposition $n a$ n'est pas pour autant moins développé.

\section{CONCLUSION}

Les recherches récentes que j'ai eu l'occasion de mener sur le nashta et le pomaque dans le cadre du marquage différentiel de l'objet m'ont permis de revenir sur son importance comme phénomène syntaxique pour l'ensemble slave nommé bulgaro-macédonien. On ne peut qu'être d'accord avec Kortman (2004) pour dire que l'étude des variétés orales non-standardisées permet de donner des descriptions typologiques plus adéquates.

On aurait par conséquent 5 cas de figure en synchronie :

a) les variétés modernes qui emploient la même unité casuelle - $a$ qu'on retrouve dans les textes écrits du vieux-slave (pomaque);

b) les variétés modernes qui emploient l'unité casuelle $-a$ et la préposition na (Struga, Ohrid, cf. Markovik 2007);

c) les variétés modernes qui emploient uniquement la préposition na (nashta);

d) les variétés qui ne distinguent pas les objets animés et inanimés en fonction de l'humanitude, soit parce qu'ils ont perdu les moyens, soit parce qu'ils ne les ont jamais développé (hypothèse probable mais jamais défendue à ma connaissance) ;

e) les variétés modernes qui ont emprunté une préposition, ex. pi (Mladenov 1993).

L'approche unifiée du marquage différentiel de l'objet, exprimé par une unité casuelle ou préposée, remet à l'ordre du jour l'argument diachronique. Tel est incontestablement le cas en pomaque, puisque les moyens d'expression, via une unité casuelle $-a$, sont identiques à ceux attestés en vieux-slave. Même si les emplois sont plus restreints qu'en nashta, on doit noter des emplois avec des noms propres féminins au singulier, fait non-attesté en vieux-slave ou dans les autres langues slaves, et signalant le caractère novateur d'un parler considéré traditionnellement comme 'archaïque' (cf. aussi Adamou à paraître 2009).

L'emploi du fonctionnel $n a$ en nashta pour l'objet direct humain est bien plus développé. J'ai proposé que son expression par une préposition devrait être considérée comme le résultat du passage d'un système casuel à un système analytique. Cette analyse est soutenue par la similarité des emplois observés en vieux-slave et dans les variétés contemporaines, par l'importance de la distinction humain $v s$ non-humain en vieux-slave, ainsi que par la faiblesse des arguments explicatifs liés au contact de langues. Malheureusement, les variétés orales n'étant 
pas écrites, nous ne disposons pas de textes qui pourraient permettre à dater avec précision le début de ce phénomène. 
Abréviations

\begin{tabular}{|c|c|c|c|}
\hline $\mathrm{ACC}$ & accusatif & NEG & négation \\
\hline ALL/DUR & allatif/duratif & NOM & nominatif \\
\hline AOR & aoriste & PART.ENONC & particule énonciative \\
\hline AUX & auxiliaire & POSS & possessif \\
\hline DAT & datif & PRF & parfait \\
\hline DEF & défini & P.PRF & plus-que-parfait \\
\hline DEF.E & défini deixis éloignée & PL & pluriel \\
\hline $\mathrm{F}$ & féminin & SG & singulier \\
\hline IMP & impératif & SUB & subordonnant \\
\hline IMPF & imparfait & VOL & volonté \\
\hline INT & intention & 2PART & deuxième participant \\
\hline LOC/PONC & locatif/ponctuel & $1 \mathrm{SG}, 2 \mathrm{SG}, 3 \mathrm{SG}$ & 1ère, 2ème, 3ème \\
\hline M & masculin & \multicolumn{2}{|c|}{ personne du singulier } \\
\hline MOD & modalité & 1PL, 2PL, 3PL & 1ère, 2ème, 3ème \\
\hline $\mathrm{N}$ & neutre & \multicolumn{2}{|c|}{ personne du pluriel } \\
\hline & & \multicolumn{2}{|c|}{$\Delta$ différentialité } \\
\hline
\end{tabular}




\section{NOTICE BIBLIOGRAPHIQUE}

ADAMOU, Evangelia, 2006 : Le nashta. Description d'un parler slave de Grèce en voie de disparition, Muenchen, Lincom.

- à paraître (2009) : Deixis and temporal subordinators in Pomak (Slavic, Greece), In I. Bril (ed.), Clause-Hierarchy and Clause-Linking: The Syntax and Pragmatics Interface, Amsterdam/Philadelphia, Benjamins, 18 p.

ADAMOU, Evangelia \& DRETTAS, Georges, 2008 : Slave, In E. Adamou (éd.),

Le patrimoine plurilingue de la Grèce. Le nom des langues II, Leuven, Peeters, p. 107-132.

ASENOVA, Petya \& ALEKSOVA, Vassilka, 2008: L'aspect balkanique de la nota accusativi personalis, Zeitschrift für Balkanologie, 44/1, Wiesbaden, Harrassowitz Verlag, p. 1-23

BARA, Maria, \& KAHL, Thede \& SOBOLEV, Andrei, 2005: Die Südaromunische Mundart von Turia (Pindos). Syntax, Lexik, Ethnolinguistik, Texte, Muenchen, Biblion Verlag.

BEIS, Stamatis, 2000 : Le parler aroumain de Metsovo. Description d'une langue en voie de disparition, thèse de doctorat, Université Paris V - René Descartes.

BOSSONG, Georges, 1985 : Empirische Universalienforschung. Differentielle Objektmarkierung in den neuiranischen Sprachen, Tübingen, Gunter Narr.

- 1998 : Le marquage différentiel de l'objet dans les langues d'Europe, In Feuillet

J. (ed), Actance et valence dans les langues de l'Europe, Berlin, Mouton de Gruyter, p. 193-258.

BREU, Walter, 2005 : Il sistema degli articoli nello slavo-molisano: eccezione a un universale tipologico, W. Breu (ed.), L'influsso dell'italiano sulla grammatica delle lingue minoritarie, Rende, Universita della Calabria, p. 111-140.

CYXUN, Gennadij, 1981: Tipologičeskie problemy balkanoslavjanskogo jazykovogo areala, Minsk, Nauka i texnika. 
DRETTAS, Georges, 1990: Le dialecte bulgaro-macédonien de Xr. (Edhessa, Grèce). Questions de typologie, Bulletin de la Société de Linguistique de Paris, 85/1, p. 227-265.

GIANNELI, Cirio \& VAILLANT, André, 1958: Un lexique macédonien du $16^{e}$ siècle, Paris, Institut d'études slaves.

GOLAB, Zbigniew, 1984 : The Arumanian dialect of Kruševo, Skopje, MANU.

HEINE, Bernd, \& CLAUDI, Ulrike \& HÜNNEMEYER, Friederike, 1991 : Grammaticalization: a conceptual framework, Chicago, The University of Chicago Press.

HEINE, Bernd \& KUTEVA Tania, 2005 : Language contact and grammatical change, Cambridge, Cambridge University Press.

HUNTLEY, David, 1993 : Old Church Slavonic, In B. Comrie, G. Corbett (eds), The Slavonic languages, London / New York, Routledge, p. 125-187.

JANSE, Mark, 2004 : Animacy, Definiteness and Case in Cappadocian and other Asia Minor Greek Dialects, Journal of Greek Linguistics, 5, p. 3-26.

KARAKASIDOU, Anastasia, 2000 : Fields of wheat, hills of blood, University of

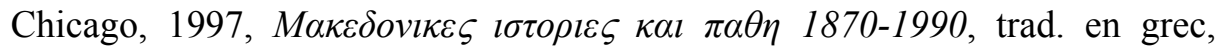
Athènes, Editions Odysseas.

KOKKA, Maria, 1992 : Texte aromâne din comuna Nea-Zoi, Jud. Tricala (Grecia), Bucureşti.

KOKKAS, Nikos, 2004 : Uchem so Pomatsko, Xanthi, Pakethra.

KONESKI, Blaze, VIDOESKI, Bozidar \& JASAR-NASTEVA, O., 1968 : Distribution des balkanismes en macédonien, Actes du premier congrès international des études balkaniques et sud-est européennes, Vol. 6, Linguistique, Sofia, 1966, p. 517-546.

KORTMAN, Bernd, 2004 : Dialectology meets typology, Berlin, Mouton de Gruyter. 
KULIKOV, Leonid, 2006 : Case systems in a diachronic perspective: A typological sketch, In Kulikov, Malchukov, de Swart (eds), Case, Valency and Transitivity, Amsterdam, Benjamins, p. 23-48.

LAZARD, Gilbert, 1984 : Actance variations and categories of the object, In Plank F. (ed), Objects. Towards a theory of grammatical relations, London, Academic Press, p. 269-292.

- 1994 : L'actance, Paris, PUF.

- 2001 : Le marquage différentiel de l'objet, In Haspelmath, König, Oesterreicher, Raible (eds), Language Typology and Language Universals, vol. 2, Berlin / New York, Mouton de Gruyter, 873-885.

LEMERLE, Pierre, 1979 t. 1, 1981 t. 2 : Les plus anciens recueils des miracles de Saint Démétrius, Paris, CNRS.

LINDSTEDT, Jouko, SPASOV, Ljudmil \& NUORLUOTO, Juhani, (eds) 2008 : The Konikovo Gospel, Helsinki, Societas Scientiarum Fennica.

MARKOVIK, Marjan, 2007: Aromanskiot i makedonskiot govor od ohridskostrushkiot region, Skopje, MANY.

MATRAS, Yaron \& SAKEL, Jeanette, 2007 : Investigating the mechanisms of pattern replication in language convergence, Studies in Language, 31/4, p. 829865.

MAZON, André \& VAILLANT, André, 1938: Evangéliaire de Kulakia, Paris, Institut d'études slaves.

MEILLET, Antoine, 1897 : Recherches sur l'emploi du génitif-accusatif en vieux slave, Paris, E. Bouillon.

MILETIC, Ljubomir, 1932: Iz života na bălgarite vo Solynsko, Makedonski pregled, VIII/1, p. 65-134.

MLADENOV, Maxim, 1993 : Bălgarskite govori v Rumŭnija, Sofia, Bălgarskata akademija na naukite.

OIKONOMIDES, Nicolas, (éd.) 1968 : Actes de Dionysiou, Lethielleux, Paris. 
PILOT-RAICHOOR, Christiane, 1994 : L'objet en badaga, Bulletin de la Société de Linguistique de Paris, 89/1, p. 359-397.

POTTIER, Bernard, 1968 : L'emploi de la préposition $a$ devant l'objet en espagnol, Bulletin de la Société de Linguistique de Paris, 63/1, p. 83-95.

SOBOLEV, Andrei, 2008 : On some Aromanian grammatical patterns in the Balkan Slavonic dialects, In The Romance Balkans, Collection of papers presented at the International Conference The Romance Balkans, Belgrade, Institut des études balkaniques, p. 113-121.

Tabula Imperii Romani K 341976 : Union académique internationale, Slovenska Akademija Znanosti, Ljubliana.

TOPOLINJSKA, Zuzana, 1995: Makedonskite dijalekti vo Egejska makedonja, Skopje, Makedonska akademija na naukite i umetnostite.

THOMASON, Sarah, 2001: Linguistic areas and language history, Haspelmath M., Koenig E., Oesterreicher W., Raible W. (eds.), Language typology and language universals, Sprachtypologie und sprachliche Universalien: An international handbook, Berlin / New York, Walter de Gruyter.

TRIFON, Nicolas, 2005 : Les Aroumains : un peuple qui s'en va, Paris, Acratie.

VAILLANT, André, 1964 : Manuel du vieux slave, tome 1, Paris, Institut d'études slaves.

WEINREICH, Uriel, 1953 [1966] : Languages in contact - findings and problems, The Hague, Mouton. 
RESUME. - The differential object marking (Bossong 1985; Lazard 2001) is a common typological phenomenon cross-linguistically. This framework when applied on South-Slavic data (Adamou 2006) throws a new light: 1. It allows a unified analysis of a common syntactic phenomenon expressed by various means: a case ("genitive-accusative" as in Pomak), an adposition (as na in Nashta), or both (e.g. Ohrid, Struga Macedonian) 2. It allows to establish a link between this phenomenon in contemporary varieties and in Old Church Slavonic (genitiveaccusative, see Meillet 1897). 3. This approach weakens the language contact hypothesis that has been claimed in order to understand "the direct object construction with the preposition $n a$ " (Koneski, Vidoeski \& Jašar-Nasteva 1968; Cyxun 1981; Topolinjska 1995; Markovik 2007; Sobolev 2008). A hypothesis furthermore fragile when we apply the methods of language contact studies (Thomason 2001; Matras \& Sakel 2007). This analysis is illustrated by first-hand data on two Slavic varieties spoken in Greece (Nashta, Pomak).

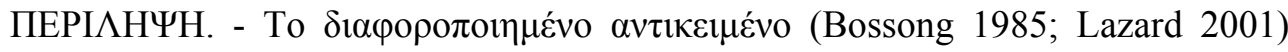

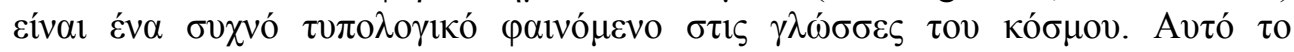

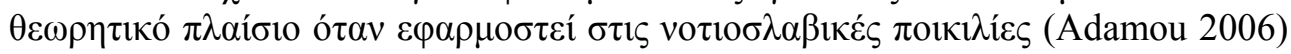

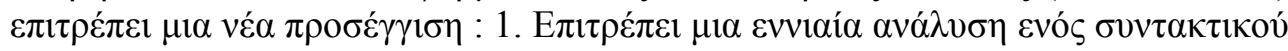

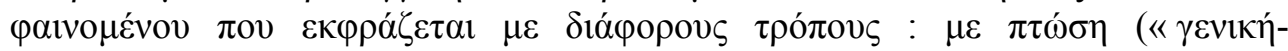

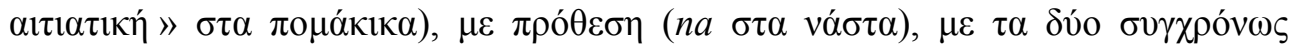

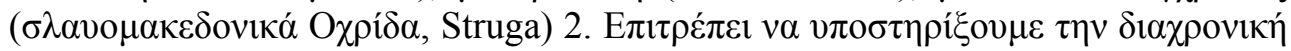

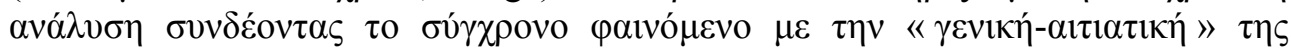

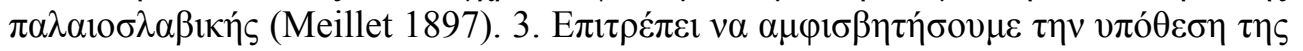

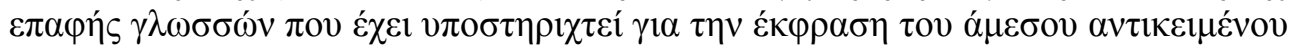

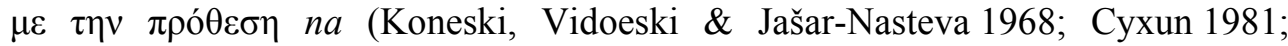

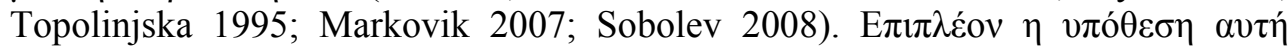

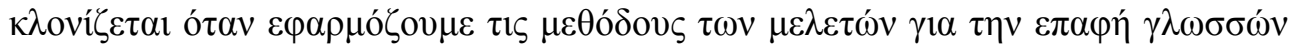

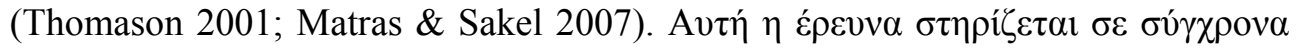

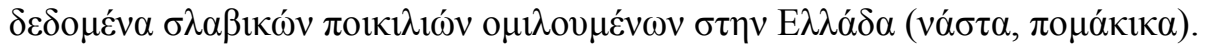

